\title{
Enterobacter cloacae septicaemia complicating rotavirus gastroenteritis: a case report
}

\author{
F.N. Öz, ${ }^{1}$ S.B. Koca, ${ }^{2}$ G. Tanır, ${ }^{1}$ D. Çiçek, ${ }^{2}$ M. Acar ${ }^{2}$ and P. Zorlu ${ }^{2}$
}

\section{Introduction}

Rotavirus infections are a common cause of gastroenteritis in infants and young children worldwide. Although rotavirus gastroenteritis is usually self-limiting in healthy infants, approximately $33 \%$ of cases of severe diarrhoea require hospitalization (1). Small intestinal epithelium dysfunction during rotavirus gastroenteritis is well-documented. Enteric Gram-negative bacteraemia as a complication of rotavirus-associated gastroenteritis has been reported in healthy children (2). It is believed that steroid treatment for any purpose may increase the risk of invasion of the mucosa by endogenous enteric bacterial flora and gastrointestinal mucosal damage via rotavirus infection. There are only 2 reported cases of secondary enteric Gram-negative bacteraemia in infants with rotavirus infection who were treated with corticosteroids for panhypopituitarism and congenital adrenal hyperplasia (3). In this paper we report the case of a patient with nosocomial rotavirus-associated gastroenteritis complicated by Enterobacter cloacae bacteraemia who was treated with adrenocorticotrophic hormone (ACTH) for infantile spasm.

\section{Case report}

A 9-month-old female child with infantile spasm was admitted to our hospital in November 2012, to regulate her uncontrolled afebrile seizures. She was born with mild perinatal asphyxia at 28 weeks' gestation by vaginal delivery after a twin pregnancy. She had been diagnosed with infantile spasm at 7 months of age and ACTH therapy had been initiated at that time.

Her physical examination at admission revealed the following findings: blood pressure $90 / 60 \mathrm{mmHg}$, pulse rate 120 beats/min., respiratory rate 28 breaths/min. and body temperature $36.4{ }^{\circ} \mathrm{C}$. Neurological examination showed developmental delay, mild hypotonia and increased leg muscle tone. The remainder of the physical findings were normal. On the 6th day of hospitalization, the patient developed watery diarrhoea. Microscopic analysis of the stool was normal and bacterial culture was negative. Rotavirus antigen was detected in the stool specimen by the $\mathrm{ABON}^{\circ}$ immunochromatographic test (Abon Biopharm Ltd).

Intravenous fluid therapy was given to the patient. Two days later, on day 8 of her hospitalization, the patient showed deterioration of clinical signs, with lethargy, fever $\left(39^{\circ} \mathrm{C}\right)$, tachycardia (170 beats/min.) and tachypnoea (50 breaths/min.). Laboratory findings at that time were as follows: haemoglobin $11 \mathrm{~g} / \mathrm{dL}$, white bloodcell count $15,900 / \mathrm{mm}^{3}$ with $70 \%$ neutrophils, platelets $87,000 / \mathrm{mm}^{3}$, C reactive protein (CRP) $118 \mathrm{mg} / \mathrm{dL}$, prothrombin time (PT) $20 \mathrm{~s}$, activated partial thromboplastin time (aPTT) $54 \mathrm{~s}$, D-dimer $2.7 \mathrm{mg} / \mathrm{L}$ (reference range: $0-0.5 \mathrm{mg} / \mathrm{L}$ ) and fibrinogen $310 \mathrm{mg} / \mathrm{dL}$. Arterial blood gas analysis showed $\mathrm{pH} 7.3, \mathrm{HCO}_{3} 7.5 \mathrm{mmol} / \mathrm{L}$, $\mathrm{pO}_{2} 110 \mathrm{mmHg}, \mathrm{pCO}_{2} 25 \mathrm{mmHg}$ and base excess 16. Serum electrolytes, liver and renal function tests and urine and stool microscopic analyses were normal. Blood and stool cultures were obtained.

Clinical sepsis and disseminated intravascular coagulation were diagnosed based on the presence of leukocytosis, thrombocytopaenia, metabolic acidosis, increased serum CRP level, prolonged PT, PTT and increased level of D-dimer, in addition to severe inflammatory response syndrome findings.

Empirical meropenem treatment and fresh-frozen plasma infusions were commenced. Abdominal ultrasonography showed a small amount of fluid retention in the intestinal loops. The blood culture of the patient at the time of clinical deterioration (on day 8) yielded E. cloaca. Antibiotic sensitivity tests revealed that the organism was sensitive to ceftriaxone, meropenem, amikacin, ciprofloxacin, but resistant to amoxicillin-clavulanic acid and cefuroxime axetil. Nosocomial rotavirus gastroenteritis, E. cloacae septicaemia and disseminated intravascular coagulation were identified as the final diagnoses of the patient.

Resolution of fever and clinical improvement occurred within 48 hours of antibiotic treatment. Subsequently, laboratory findings returned to normal and control blood culture was negative. 
The patient was discharged after a 14day course of meropenem treatment and an uneventful recovery.

\section{Discussion}

Acute gastroenteritis is a common disease in children, especially during the first year of life, and rotavirus is the major etiologic agent of acute gastroenteritis (1). Nosocomial rotavirus infection is an important and well-known problem in hospitalized, unvaccinated infants. Rotavirus spreads efficiently via a fecal-oral route, and outbreaks are common in children's hospitals and child-care centres. The virus is shed in stools at very high concentrations before and 4 days after the clinical illness (4). Patients and health-care workers with asymptomatic rotavirus infection may also spread the disease. According to our hospital infection surveillance reports, there was no other case in the ward at November 2012. Nosocomial rotavirus gastroenteritis was diagnosed because the patient's diarrhoea occurred after 48 hours of hospitalization.

It is known that rapid detection of rotavirus antigen in fresh stool is easily and accurately accomplished, with excellent specificity and sensitivity, using commercially available latex agglutination assay, enzyme-linked immunosorbent assays and chromatographic immunoassays (5). The diagnosis of rotavirus gastroenteritis in the present patient was established using a rapid chromatographic immunoassay that uses the double-antibody sandwich method for the qualitative detection of rotavirus in a stool specimen.

Secondary bacterial complications of rotavirus infection have been reported in children. In one study, 27 (13.0\%) of 207 children with nontyphoid Salmonella gastroenteritis were found to be co-infected with rotavirus. Furthermore, complications of non-typhoid Salmonella bacteraemia were more frequent in patients coinfected with rotavirus (6). Recently, a comprehensive review included 20 paediatric cases of septicaemia after rotavirus gastroenteritis was reported. Only 2 of these 20 patients were over 3 years of age (2). The present case of nosocomial rotavirus gastroenteritis complicated with E. cloacae septicaemia was also in an infant. Rotavirus has been shown to cause intestinal epithelial metaplasia and dysfunction. Rotavirus-infected enterocytes are more vulnerable to bacterial invasion. The primary mechanism of septicaemia developing from rotavirus infection is not fully understood, but it is thought that translocation of enteric flora across intestinal epithelium damaged by rotavirus puts a patient at risk for secondary septicaemia (7).

A previous report found that 20 paediatric cases who had septicaemia complications of rotavirus gastroenteritis had no prior intestinal disease or immunodeficiency (2). In contrast to these reports, our patient was under ACTH treatment, a potent stimulator of steroid synthesis. Two cases with congenital adrenal hyperplasia and panhypopituitarism that presented with Klebsiella pneumonia bacteraemia and rotavirus co-infection during corticosteroid treatment have been reported (3). The effects of corticosteroids on the gastrointestinal tract and their pathophysiology are not clearly understood. Delayed epithelial healing is most likely due to inhibition of prostaglandin synthesis (8). The sideeffects of ACTH are more common at higher doses and include hypertension, electrolyte imbalance, infections, hyperglycaemia, and gastric ulcers (9). It is thought that delayed healing due to steroid administration combined with gastrointestinal mucosal dysfunction due to rotavirus infection may increase the risk of invasion of the mucosa by endogenous enteric bacterial flora. The case presented here suggests that septicaemia complication of nosocomial rotavirus gastroenteritis may be especially important in the presence of ACTH treatment. The possibility of this complication should be considered in the case of fever, signs of sepsis and elevated leukocyte count, thrombocytopenia or elevated inflammatory markers while a child has rotavirus gastroenteritis. It is important to obtain blood cultures in these cases for early identification and treatment of a child developing bacterial superinfection after rotavirus. Prevention of nosocomial rotavirus infections may be an important factor to improve patient safety, especially for immunosuppressed patients. For this reason, we also emphasize the importance of infection control in hospitals and for rotavirus vaccination to prevent unnecessary hospital admissions.

Competing interests: None declared.

\section{References}

1. Parashar UD, Bresee JS, Gentsch JR, Glass RI. Rotavirus. Emerg Infect Dis. 1998 Oct-Dec;4(4):561-70. PMID:9866732

2. Scheier $\mathrm{E}$, Aviner S. Septicemia following rotavirus gastroenteritis. Isr Med Assoc J. 2013 Mar;15(3):166-9. PMID:23662380

3. Longmore DK, Batch JA, McMahon SK, Conwell LS. Klebsiella pneumoniae bacteraemia complicating rotavirus gastroenteritis in two infants with glucocorticoid deficiency. J Pediatr Endocrinol Metab. 2010 Mar;23(3):293-5. PMID:20480730
4. Bass DM. Rotaviruses, caliciviruses, and astroviruses. In: Kliegman RM, Behrman RE, Jenson HB, editors. Nelson textbook of pediatrics. 19th ed. Philadelphia: Elsevier; 2011:1134-7.

5. Costello M, Sabatini LM, Yungbluth M. Viral infections. McPherson RA, Pincus MR, editors. Henry's clinical diagnosis and management by laboratory methods. Philadelphia: Elsevier; 2011:1038-64. 
6. Hung TY, Liu MC, Hsu CF, Lin YC. Rotavirus infection increases the risk of bacteremia in children with nontyphoid Salmonella gastroenteritis. Eur J Clin Microbiol Infect Dis. 2009 Apr;28(4):425-8. PMID:18949496

7. Lowenthal A, Livni G, Amir J, Samra Z, Ashkenazi S. Secondary bacteremia after rotavirus gastroenteritis in infancy. Pediatrics. 2006 Jan;117(1):224-6. PMID:16396884
8. Carpani de Kaski M, Rentsch R, Levi S, Hodgson HJ. Corticosteroids reduce regenerative repair of epithelium in experimental gastric ulcers. Gut. 1995 Nov;37(5):613-6. PMID:8549934

9. Mikati MA. Treatment of seizures and epilepsy. In: Kliegman RM, Behrman RE, Jenson HB, editors. Nelson textbook of pediatrics. 18th ed. Philadelphia: Saunders Elsevier; 2007:1605-18 